\title{
Accretion-outflow connection in the outliers of the "universal" radio/X-ray correlation
}

\author{
M. Coriat,${ }^{1} \dagger$ S. Corbel,${ }^{1}$ L. Prat,${ }^{1}$ J. C. A. Miller-Jones,${ }^{2}$ D. Cseh,${ }^{1}$ \\ A. K. Tzioumis,${ }^{3}$ C. Brocksopp,${ }^{4}$ J. Rodriguez, ${ }^{1}$ R. P. Fender ${ }^{5}$ \\ and G. R. Sivakoff ${ }^{6}$ \\ ${ }^{1}$ Laboratoire AIM, CEA-IRFU/CNRS/Université Paris Diderot, \\ CEA Saclay, F-91191 Gif-sur-Yvette, France \\ email: mickael.coriat@cea.fr, stephane.corbel@cea.fr, lionel.prat@cea.fr, \\ david.cseh@cea.fr, jerome.rodriguez@cea.fr \\ ${ }^{2}$ NRAO Headquarters, 520 Edgemont Road, Charlottesville, VA 22903, USA \\ email: james.miller-jones@curtin.edu.au \\ ${ }^{3}$ Australia Telescope National Facility, CSIRO, P.O. Box 76, Epping, NSW 1710, Australia \\ email: tasso.tzioumis@csiro.au \\ ${ }^{4}$ Mullard Space Science Laboratory, University College London, \\ Holmbury St. Mary, Dorking, Surrey RH5 6NT, UK \\ email: cb4@mssl.ucl.ac.uk \\ ${ }^{5}$ School of Physics and Astronomy, University of Southampton, Southampton SO17 1BJ, UK \\ email: $r$.fender@soton.ac.uk \\ ${ }^{6}$ Department of Astronomy, University of Virgina, P.O. Box 400325, \\ Charlottesville, VA 22904-4325, USA \\ email: grs8g@virginia.edu
}

\begin{abstract}
In recent years, numerous efforts have been devoted to unravel the connection between accretion flow and jets in accreting compact objects. Here we report new constraints on these issues, through the long term study of the radio and X-ray behaviour of the black hole candidate $\mathrm{H} 1743-322$. This source is known to be one of the "outliers" of the universal radio/X-ray correlation, i.e. a group of stellar mass accreting black holes displaying fainter radio emission for a given X-ray luminosity, than expected from the correlation. In this work we find, at high X-ray luminosity in the hard state, a tight radio/X-ray correlation with an unusual steep slope of $b=1.38 \pm 0.03$. This correlation then breaks below $\sim 5 \times 10^{-3} L_{\mathrm{Edd}}\left(M / 10 M_{\odot}\right)^{-1}$ in $\mathrm{X}$-rays and becomes shallower. When compared with radio/X-ray data from other black hole $\mathrm{X}$-ray binaries, we see that the deviant points of $\mathrm{H} 1743-322$ join the universal correlation and seem to follow it at low luminosity. Based on these results, we investigate several hypotheses that could explain both the $b \sim 1.4$ slope and the transition toward the universal correlation.
\end{abstract}

Keywords. black hole physics; X-rays: binaries; galaxies: jets; accretion, accretion disks; radiation mechanisms: nonthermal

\section{Introduction}

Observations of several black hole X-ray binaries (BHXBs) have brought evidence that a strong correlation exists, during the hard X-ray state, between radio and X-ray emissions (e.g. Corbel et al. 2003; Gallo et al. 2003). This connection takes the form of a non-linear flux correlation, $F_{\mathrm{Rad}} \propto F_{\mathrm{X}}^{b}$, where $F_{\mathrm{Rad}}$ is the radio flux density, $F_{\mathrm{X}}$ is the X-ray flux and $b \sim 0.5-0.7$. This correlation highlight the strong link between the

$\dagger$ Present address: School of Physics and Astronomy, University of Southampton, Southampton SO17 1BJ, UK. email: m.coriat@soton.ac.uk 
compact jets and the accretion flow (disk and/or corona). This radio/X-ray correlation, initially established for the source GX 339-4, has been extended to other galactic black holes (mainly V404 Cyg, Gallo et al. 2003; Corbel et al. 2008) and even active galactic nuclei (Merloni et al. 2003; Falcke et al. 2004; Körding et al. 2006).

However in the following years, a few galactic black hole candidates (BHCs) have been found to lie outside the scatter of the original radio/X-ray correlation, (e.g. XTE J1720-318, Brocksopp et al. 2005; XTE J1650-500, Corbel et al. 2004; IGR J17497-2821, Rodriguez et al. 2007; Swift J1753.5-0127, Cadolle Bel et al. 2007, Soleri et al. 2010) thus either increasing its scatter, or challenging the universality of the correlation itself. For a given X-ray luminosity, these outliers show a radio luminosity fainter than excepted from the correlation (and thus are sometimes dubbed "radio-quiet" black holes). However, for most of these outliers, there are no radio measurements available at low X-ray luminosities. Therefore we do not know whether they remain "under luminous" in radio at low accretion rates. The current lack of data also prevents to precisely measure the slope of the correlation (if any) for the outliers. It is thus unclear if the outliers follow a correlation similar to the "standard" BHXBs but with a lower normalisation or if their inflow/outflow connection is intrinsically different. Moreover, we do not know if their behaviour is recurrent over several outbursts.

These are the issues that motivated the work presented here on the outlier H 1743-322. This source was discovered during a bright outburst in 1977 (Kaluzienski \& Holt 1977). Since then, it underwent six outbursts between 2003 and 2010. All were followed by various X-ray satellites and ground-based radio observatories, providing a comprehensive set of data to study the accretion/ejection coupling in this source. The purpose of this work is to investigate this connection in detail through the study of the radio/X-ray correlation over the 6 outbursts. Consequently, we analyse almost all available radio data covering these outbursts, together with all the X-ray data from the Rossi X-ray Timing Explorer ( $R X T E)$ instruments. The sequence of observations and data reduction processes are detailed in Coriat et al. (submitted). In the following we very briefly present the main results of this study.

\section{Results and discussion}

Figure 1 shows the radio $8.5 \mathrm{GHz}$ luminosity against the X-ray 3-9 keV luminosity of $\mathrm{H} 1743-322$ in the hard states of the 6 outbursts. Above $\sim 10^{36} \mathrm{erg} \mathrm{s}^{-1}$ in the $3-9 \mathrm{keV}$ band, we note a clear correlation over one orders of magnitude. We fit the data with a powerlaw of the form $L_{\mathrm{Rad}} \propto L_{\mathrm{X}}^{b}$, where $L_{\mathrm{Rad}}$ is the radio luminosity and $L_{\mathrm{X}}$ is the $\mathrm{X}$-ray luminosity. We obtain $b=1.38 \pm 0.03$. This correlation index clearly differs from the one usually observed for BHXBs (i.e. 0.5-0.7). We also note that some data points clearly depart from the correlation below $\sim 2 \times 10^{36} \mathrm{erg} \mathrm{s}^{-1}$ in X-ray. This could indicate a significant evolution of the inflow - outflow connection when the source reaches low luminosities. To locate H 1743-322 with regards to the universal radio/X-ray correlation of black holes and neutron stars X-ray binaries, we also plot on Fig. 1 the data from the black holes GX 339-4 (Corbel et al., in prep), V404 Cyg (Gallo et al. 2003; Corbel et al. 2008), Swift J1753.5-0127 (Soleri et al. 2010) and GRO J1655-40 and from the neutron stars Aql X-1 (Tudose et al. 2009; Miller-Jones et al. 2010) and 4U 1728-34 (Migliari \& Fender 2006). Fig. 1 shows that H 1743-322 lies significantly below the correlation for BHXBs but is still more "radio-loud" than neutron stars. As noted by Soleri et al. (2010), the data from Swift J1753.5-0127 are consistent with a correlation index of 1.4. In addition, we note the deviant points at low luminosity that join the standard correlation driven by GX 339-4 and V404 Cyg, and seem to follow it (cf. 


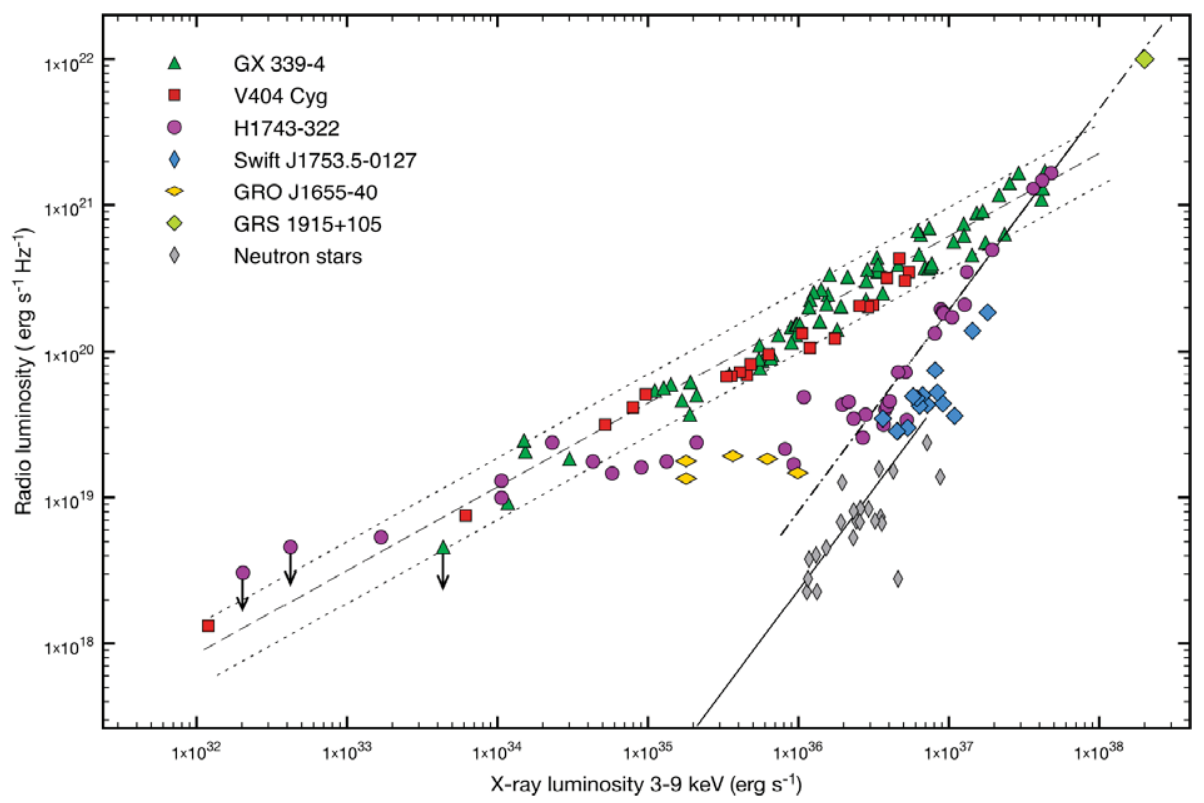

Figure 1. Radio $8.5 \mathrm{GHz}$ luminosity against X-ray $3-9 \mathrm{keV}$ luminosity from the hard state BHCs H 1743-322, GX 339-4, V404 Cyg, Swift J1753.5-0127, GRO J1655-40 and the hard state neutron stars Aql X-1 and 4U1728-34. A representative data point of GRS $1915+105$ in the plateau state is also plotted. The dashed-dotted line is the fit to the high luminosity data of H 1743-322 with a function of the form $L_{\mathrm{Rad}} \propto L_{\mathrm{X}}^{b}$, with $b \sim 1.4$. The dashed line is the fit to the GX 339-4 and V404 Cyg data with $b \sim 0.6$. The dotted lines define the scattering around the fit. The solid line is the fit to the neutron stars data with $b \sim 1.4$

points below $2 \times 10^{34} \mathrm{erg} \mathrm{s}^{-1}$ ). We also note that GRO J1655-40 seems to follow the same deviation. This supports the idea of a significant transition in the coupling between the jets and the X-ray emitting component. To complete this figure, we add a "representative" point of GRS $1915+105$ in the plateau state (Fender et al. 2010). We see that it lies on the extrapolation of the correlation of the outliers. This could be purely fortuitous but given the peculiar behaviour of this source, we thought it was worth highlighting its possible association with the outliers.

As mentioned in the introduction, some of the numerous questions associated with the outliers are whether they follow the same correlation as the other BHXBs but with a lower normalisation, and whether they remain under luminous in radio at low accretion rates. In the case of $\mathrm{H} 1743-322$, we obtain a correlation coefficient of $b=1.38 \pm 0.03$. This is the first precise measurement of the radio/X-ray correlation of an outlier. If $\mathrm{H} 1743-322$ is representative of these radio-quiet Galactic black holes, our results suggest that their radio sub-luminosity is due to a different correlation coefficient rather than a different normalisation constant. Concerning the behaviour at low luminosity, the data suggest that the outliers do not remain sub-luminous in radio but rather seem to dovetail with the "standard" correlation.

As we show in Coriat et al. (submitted), one of the possible interpretation of these results is to consider that the difference between the standards BHXBs and the outliers arise from the radiative efficiency of their accretion flow. It is usually accepted that accreting black holes in the hard state display a radiatively inefficient inner accretion flow with $L_{X} \propto \dot{M}^{2-3}$. If we consider that the outlier rather display a radiatively efficient accretion flow (with $L_{X} \propto \dot{M}$ ) we can reproduce the observed correlation. However, below 


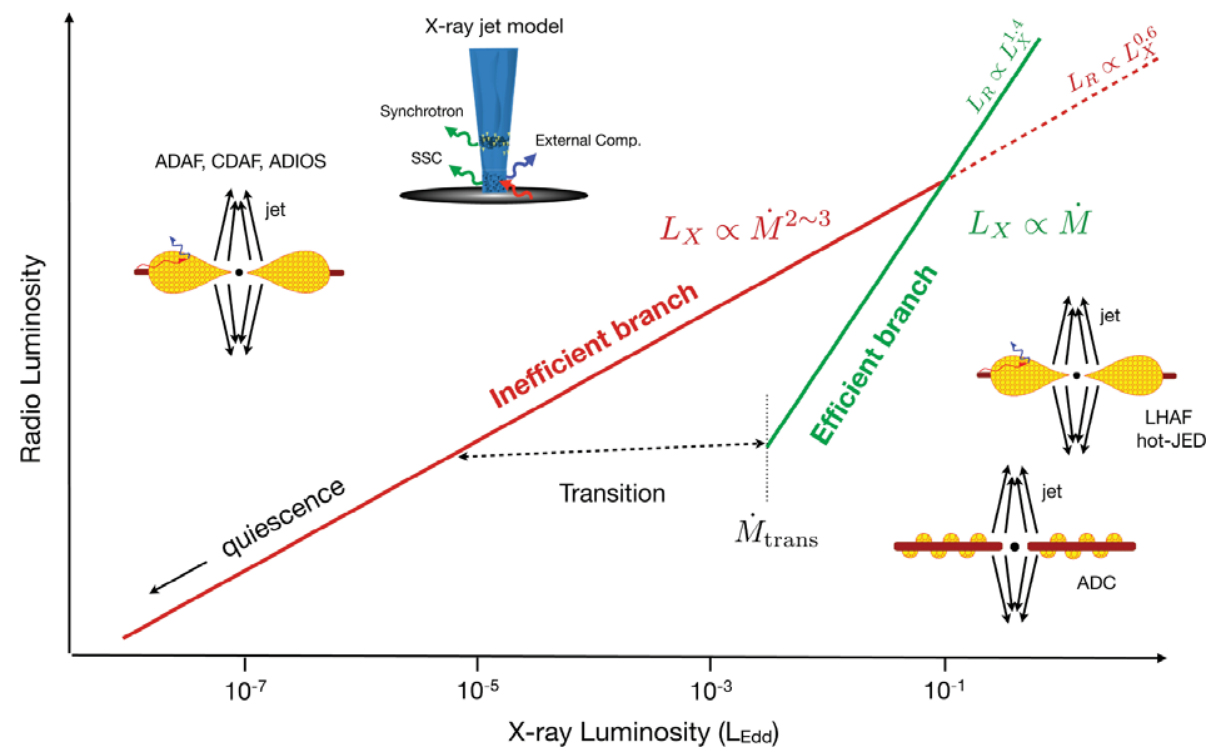

Figure 2. A schematic drawing of the global radio/X-ray correlation for galactic black holes. The X-ray luminosity is expressed in terms of the Eddington luminosity for a $10 M_{\odot}$ black hole. On this figure, we illustrate the case where the steep correlation of the outliers is a consequence of the coupling between a radiatively efficient accretion flow and a steady compact jet. We can then distinguish two branches in the radio/X-ray diagram of BHXBs, according to the efficiency of the accretion flow and the consequent scaling of the X-ray luminosity with the mass accretion rate. We also illustrate the possibility of a transition between branches below a critical accretion rate $\dot{M}_{\text {trans }}$. Some examples of models (figures adapted from Markoff et al. 2005 and M. Nowak) that could explain the accretion/ejection properties of each branch are also represented.

a critical accretion rate $\dot{M}_{\text {trans }}$, the flow would have to become radiatively inefficient to account for the transition at low luminosity. This idea is summarised by the sketch of Fig. 2. Due to the limited number of pages, we refer the interested reader to Coriat et al. (submitted) for a more comprehensive and detailed discussion and alternative interpretations.

\section{References}

Brocksopp, C., Corbel, S., Fender, R. P., et al. 2005, MNRAS, 356, 125

Cadolle Bel, M., Ribó, M., Rodriguez, J., et al. 2007, ApJ , 659, 549

Corbel, S., Fender, R. P., Tomsick, J. A., Tzioumis, A. K., \& Tingay, S. 2004, ApJ , 617, 1272

Corbel, S., Körding, E., \& Kaaret, P. 2008, MNRAS, 389, 1697

Corbel, S., Nowak, M. A., Fender, R. P., Tzioumis, A. K., \& Markoff, S. 2003, A $\mathscr{E} A$, 400, 1007

Falcke, H., Körding, E., \& Markoff, S. 2004, A\&A , 414, 895

Fender, R., Gallo, E., \& Russell, D. 2010, ArXiv e-prints

Gallo, E., Fender, R. P., \& Pooley, G. G. 2003, MNRAS, 344, 60

Kaluzienski, L. J. \& Holt, S. S. 1977, IAU Circ., 3099, 3

Körding, E., Falcke, H., \& Corbel, S. 2006, A\&A , 456, 439

Markoff, S., Nowak, M. A., \& Wilms, J. 2005, ApJ, 635, 1203

Merloni, A., Heinz, S., \& di Matteo, T. 2003, MNRAS, 345, 1057

Migliari, S. \& Fender, R. P. 2006, MNRAS, 366, 79

Miller-Jones, J. C. A., Sivakoff, G. R., Altamirano, D., et al. 2010, ApJL , 716, L109

Rodriguez, J., Cadolle Bel, M., Tomsick, J. A., et al. 2007, ApJL , 655, L97

Soleri, P., Fender, R. P., Tudose, V., et al. 2010, MNRAS, in press (arXiv:1004.1066)

Tudose, V., Fender, R. P., Linares, M., Maitra, D., \& van der Klis, M. 2009, MNRAS, 400, 2111 


\section{Discussion}

MEIER: Do you have a good idea of the type of power spectra in your RQ BHs? GRO J1655-40 and GRS 1915+105 are on your transition line and alternate correlation line, respectively, and have peculiar power spectra, flat with a cutoff at $\sim 10 \mathrm{~Hz}$.

CORIAT: Indeed, we see similar power spectra in H1743, but maily when the source is in the hard intermediate state. We see a flat part with a cutoff at around $\sim 5 \mathrm{~Hz}$ (bur it evolves between 2 and $10 \mathrm{~Hz}$ ). Around the cutoff frequency, we usually see a strong type C QPO with several harmonics. But this kind of PDS seem to be typical of the hard intermediate state.

YUAN: A comment on disk-corona model for hard X-ray emission. Observationally, the high/soft state is described by the disk, but hard the X-ray emission is very weak. Theoretically, simulations show that the corona is very weak to produce hard X-rays.

CoRIAT: From your observational argument, I don't know if it really excludes the possibility that hard X-ray emission in the hard state originates from a magnetic corona. Indeed, the accretion conditions could be different. I am not an expert in simulations of corona, but it seems that several simulations show strong hard X-ray emission from corona. So, as it is not clear whether a corona could be responsible or not or the hard X-ray emission, we just don't want to exclude this possibility. 